University of Wollongong

Research Online

Faculty of Engineering and Information

Faculty of Engineering and Information

Sciences - Papers: Part B

Sciences

2017

\title{
High efficiency and non-Richardson thermionics in three dimensional Dirac materials
}

Sunchao Huang

University of Wollongong, sh676@uowmail.edu.au

Matthew Sanderson

University of Wollongong, msanders@uow.edu.au

Yan Zhang

Capital Normal University

C Zhang

University of Wollongong, czhang@uow.edu.au

Follow this and additional works at: https://ro.uow.edu.au/eispapers1

Part of the Engineering Commons, and the Science and Technology Studies Commons

Research Online is the open access institutional repository for the University of Wollongong. For further information contact the UOW Library: research-pubs@uow.edu.au 


\title{
High efficiency and non-Richardson thermionics in three dimensional Dirac materials
}

\author{
Abstract \\ Three dimensional (3D) topological materials have a linear energy dispersion and exhibit many electronic \\ properties superior to conventional materials such as fast response times, high mobility, and chiral \\ transport. In this work, we demonstrate that 3D Dirac materials also have advantages over conventional \\ semiconductors and graphene in thermionic applications. The low emission current suffered in graphene \\ due to the vanishing density of states is enhanced by an increased group velocity in 3D Dirac materials. \\ Furthermore, the thermal energy carried by electrons in 3D Dirac materials is twice of that in conventional \\ materials with a parabolic electron energy dispersion. As a result, 3D Dirac materials have the best \\ thermal efficiency or coefficient of performance when compared to conventional semiconductors and \\ graphene. The generalized Richardson-Dushman law in 3D Dirac materials is derived. The law exhibits the \\ interplay of the reduced density of states and enhanced emission velocity.

\section{Disciplines} \\ Engineering | Science and Technology Studies

\section{Publication Details} \\ Huang, S., Sanderson, M., Zhang, Y. \& Zhang, C. (2017). High efficiency and non-Richardson thermionics in \\ three dimensional Dirac materials. Applied Physics Letters, 111 (18), 183902-1-183902-4.
}




\title{
High efficiency and non-Richardson thermionics in three dimensional Dirac materials
}

\author{
Sunchao Huang, ${ }^{1}$ Matthew Sanderson, ${ }^{1}$ Yan Zhang, ${ }^{2,3}$ and Chao Zhang ${ }^{1,3, a)}$ \\ ${ }^{1}$ School of Physics, University of Wollongong, Northfield Avenue, New South Wales 2522, Australia \\ ${ }^{2}$ Department of Physics, Capital Normal University, Beijing Key Laboratory of Metamaterials and Devices, \\ Key Laboratory of Terahertz Optoelectronics, Ministry of Education, Beijing 100048, China \\ ${ }^{3}$ Beijing Advanced Innovation Center for Imaging Technology, Beijing 100048, China
}

(Received 23 September 2017; accepted 11 October 2017; published online 30 October 2017)

\begin{abstract}
Three dimensional (3D) topological materials have a linear energy dispersion and exhibit many electronic properties superior to conventional materials such as fast response times, high mobility, and chiral transport. In this work, we demonstrate that 3D Dirac materials also have advantages over conventional semiconductors and graphene in thermionic applications. The low emission current suffered in graphene due to the vanishing density of states is enhanced by an increased group velocity in 3D Dirac materials. Furthermore, the thermal energy carried by electrons in 3D Dirac materials is twice of that in conventional materials with a parabolic electron energy dispersion. As a result, 3D Dirac materials have the best thermal efficiency or coefficient of performance when compared to conventional semiconductors and graphene. The generalized Richardson-Dushman law in 3D Dirac materials is derived. The law exhibits the interplay of the reduced density of states and enhanced emission velocity. Published by AIP Publishing. https://doi.org/10.1063/1.5006277
\end{abstract}

The power generation and cooling based on electron (or hole) transport has been continually researched for over 100 years. Solid state power generation and cooling are based on two mechanisms; thermoelectrics (TE) and thermionics. Both realize energy conversion between heat and electricity. Thermoelectric materials are characterized by the merit, defined as $Z T=S^{2} \sigma T\left(\kappa_{L}+\kappa_{e}\right)^{-1}$, where $S$ is the Seebeck coefficient, $\sigma$ is the electrical conductivity, $T$ is the temperature, and $\kappa_{L}$ (lattice) and $\kappa_{e}$ (electronic) are thermal conductivities. The relatively low thermodynamic efficiency of commonly used TE materials has prevented their use in largescale applications. Advancement of TE materials and devices requires finding materials with a high $S$, a high $\sigma$, and low $\kappa_{L}$ and $\kappa_{e}$. The bottleneck is that these quantities are intrinsically connected, as a good electrical conductor is normally a good thermal conductor, thus making further improvement of $Z T$ of bulk materials extremely difficult. Moreover, $\kappa_{L}$ of a solid cannot be reduced below its amorphous limit. ${ }^{1-4}$ Recent development in nanotechnology allows further reduction of $\kappa_{L}$ by using material design, crystal structure design ${ }^{1,2,5,6}$ and nanostructuring, ${ }^{3,7-10}$ nano-precipitation in $\mathrm{PbTe},{ }^{10}$ nano-grained $\mathrm{Bi}_{2} \mathrm{Te}_{3},{ }^{3,10}$ and liquid-like ions in $\mathrm{Zn}_{4} \mathrm{Sb}_{3}$ (Ref. 2) and $\mathrm{Cu}_{2} \mathrm{Se}^{1}$ These approaches have been the focus in thermoelectric research in the last decades and have resulted in an increase of the peak $Z T$ to 1.5 and higher. It has been demonstrated that a high band degeneracy, ${ }^{11-13}$ a low band effective mass, ${ }^{14}$ and weak scattering strength ${ }^{15}$ can lead to an increase in $Z T$.

Thermionic generators or coolers operate using an energy barrier between two materials at different temperatures to allow high-energy electrons in the hot material to flow across the barrier into the cold material, establishing an electrical current. They differ from thermoelectric devices in the way electrons travel. In thermoelectric devices, electrons travel diffusively. In thermionic devices, the barrier between the hot and cold regions of the device is shorter than the mean free path of the electrons, so electrons travel ballistically. The efficiency of both thermoelectric and conventional thermionic devices is lower than the theoretical upper limit, known as the Carnot efficiency, for all finite barrier heights. ${ }^{16}$ The basic structure of the thermionic cooler consists of two planar electrodes separated by a potential barrier, such as metal-vacuum-metal system. ${ }^{17}$

Semiconductor systems were considered as a promising candidate for thermionic cooling due to their low work functions. The science of thermionics and its potential applications in integrated cooling of electronic and optoelectronic devices have attracted considerable interest both theoretically and experimentally. The newly discovered materials with a linear energy-momentum dispersion such as graphene, ${ }^{18}$ topological materials, ${ }^{19}$ and Dirac materials $^{20}$ have attracted enormous attention in the past decade. These materials exhibit extremely high mobility, strong optical absorption, and a fast response. These properties make this class of Dirac materials a promising candidate for applications in electronics and photonics. ${ }^{21-23}$ However, it is generally expected that energy transport in these materials would be less significant because of the zero mass nature of charge carriers. In this paper, we shall demonstrate that while the thermal emission current from Dirac particles is less than that from massive carriers, the thermal efficiency and the coefficient of performance in massless thermionic device is higher compared to the massive particle based thermionic devices.

The thermionic process was originally discovered by Richardson in 1901 who proposed a law, now known as the Richardon-Dushman (RD) law, to describe the emission current density of conventional materials ${ }^{17}$

$$
J_{R}(\phi, T)=\frac{q m k_{B}^{2}}{2 \pi^{2} \hbar^{3}} T^{2} e^{-q \phi \beta}=A_{R} T^{2} e^{-q \phi \beta},
$$

a)czhang@uow.edu.au 
where $\phi$ is the surface potential, $T$ is the temperature, $q$ is the electronic charge, $m$ is the mass of electron, $k_{B}$ is the Boltzmann constant, $\hbar$ is the reduced Planck's constant, $\beta=1 /\left(k_{B} T\right)$ and $A_{R} \approx-120 \mathrm{~A} \mathrm{~cm}^{-2} \mathrm{~K}^{-2}$ is the Richardson constant. ${ }^{17}$ The conventional materials refer to those having parabolic energy-momentum dispersion.

Recently, the thermionic properties of graphene have been investigated both experimentally ${ }^{24}$ and theoretically. ${ }^{25}$ The results showed that the mass-dependent RichardsonDushman no longer holds for graphene due to the massless energy dispersion, $E=\hbar k v_{F}$, where $v_{F}$ is the Fermi velocity and $k=|\mathbf{k}|$ is the electron wavevector. A modified thermionic law was established by assuming graphene to have a linear energy-momentum dispersion within the layer and a parabolic energy-momentum dispersion in the perpendicular direction $^{25,26}$

$$
J_{G}(\phi, T)=\frac{q k_{B}^{3} T}{\pi^{2} \hbar^{3} v_{F}^{2}} T^{2} e^{-q \phi \beta}=A_{G} T^{2} e^{-q \phi \beta},
$$

where $A_{G}$ is the modified RD constant for graphene. The modified RD law is mass independent. The main difference between graphene and conventional materials is that $A_{G}$ is linearly dependent on temperature. The work function of graphene extracted by the law agrees well with results in literature. ${ }^{27,28}$ Based on the modified law, graphene-based thermionic devices were further investigated. ${ }^{26,29}$ Although the free-standing single layer graphene has high electron mobility, its thermionic emission ability is limited due to a small density of states and its emission ability would be affected by a substrate. Recently, research showed $\mathrm{Cd}_{3} \mathrm{As}_{2}{ }^{30-32}$ has an unexpectedly low thermal conductivity and extraordinary performance in thermoelectric devices. ${ }^{33}$ In the present work, we will analyse the thermionic emission and cooling based on 3D Dirac materials.

For 3D Dirac semimetals with a low energy-momentum dispersion given by $E=\hbar k v_{F}$, the number of electrons per unit volume $n(E)$ and the velocity $v_{z}$ are given by

$$
\begin{aligned}
n(E) & \approx \frac{E^{2}}{\pi^{2} \hbar^{3} v_{F}^{3}} e^{\left(E_{F}-E\right) \beta}, \\
v_{z} & =\frac{\partial H}{\partial p_{z}}=\frac{v_{F} k_{z}}{k},
\end{aligned}
$$

where $E_{F}$ is the Fermi energy. In Eq. (3), we have assumed $E-E_{F}>3 k_{B} T$, which enables us to replace the Fermi-Dirac distribution with the Maxwell-Boltzmann distribution. The assumption is reasonable if the work function $(q \phi)$ is greater than $3 k_{B} T$ (about $90 \mathrm{meV}$ ). The thermionic emission current density is calculated by

$$
J_{D}=\int q n(E) v_{z}(E) \mathrm{d} E=A_{D} T^{2} \mathrm{e}^{-q \phi \beta},
$$

with modified Richardson's coefficient

$$
A_{D}=\frac{q k_{B}^{2}}{\pi^{2} \hbar^{3} v_{F}^{2}}\left(q \phi+E_{F}+k_{B} T\right)
$$

where $J_{R}$ is mass dependent, and $J_{G}$ and $J_{D}$ are mass independent due to the massless nature of charge carriers in
Dirac systems. $J_{R}$ and $J_{G}$ have parabolic and cubic temperature dependence, respectively. The temperature dependence of the thermionic current density for conventional materials $\left(J_{R}\right)$, single graphene $\left(J_{G}\right)$, and 3D Dirac materials $\left(J_{D}\right)$ are shown in Fig. 1. $J_{G}$ is much smaller compared to the emission current from other systems so we plot $10 J_{G}$ in the figure. Figure 1 shows $J_{R}>J_{D}>J_{G}$, which indicates that conventional materials have the highest rate of thermionic emission and that single-layer graphene has the lowest rate. Comparing Eq. (5) to Eq. (2), the emission current from a 3D Dirac system is greater than that from graphene by an amount, $\Delta J=\frac{q k_{B}^{2}}{\pi^{2} \hbar^{3} v_{F}^{2}}\left(q \phi+E_{F}\right) T^{2} e^{-q \phi \beta}$. This difference has the same form as $J_{R}^{F}$ in terms of the temperature dependence. This is an intriguing result. In the model used to calculate $J_{G}$, the carriers have a linear energy dispersion in the direction perpendicular to the emission direction and a parabolic dispersion in the emission direction. The emission current is greatly reduced as compared to $J_{R}$. In 3D Dirac materials, the energy dispersion is linear in all three directions so $J_{D}$ adds an extra term on to $J_{G}$. We analyse this change by considering both the density of states and the group velocity of emitted electrons. The emission current increases with both the density of states and the emission velocity. When the inplane energy dispersion changes from parabolic to linear, the density of states is significantly reduced near the bottom of the conduction band. This is the origin of $J_{G}$ being much smaller than $J_{R}$. On the other hand, the group velocity of the emitted electrons along the emission direction, given by $\partial E / \partial k_{z}$, increases when the energy dispersion along the $k_{z}$ direction changes from a parabolic to linear momentum dependence. For a parabolic dispersion, the group velocity goes to zero as $k_{z}$ goes to zero. For a linear dispersion, the group velocity can be finite when $k_{z}$ goes to zero if $k_{x}$ and $k_{y}$ both go to zero. In the case of 3D Dirac materials, the vanishing in-plane density of states is somewhat compensated by the enhancement of the group velocity along the emission direction. Our result indicates that the materials with the highest emission current would be those with a parabolic inplane energy dispersion and linear energy dispersion along the emission direction.

Based on the above model, we consider a thermionic refrigerator made up of Dirac materials. The net charge current and the net energy current can be written as ${ }^{17}$

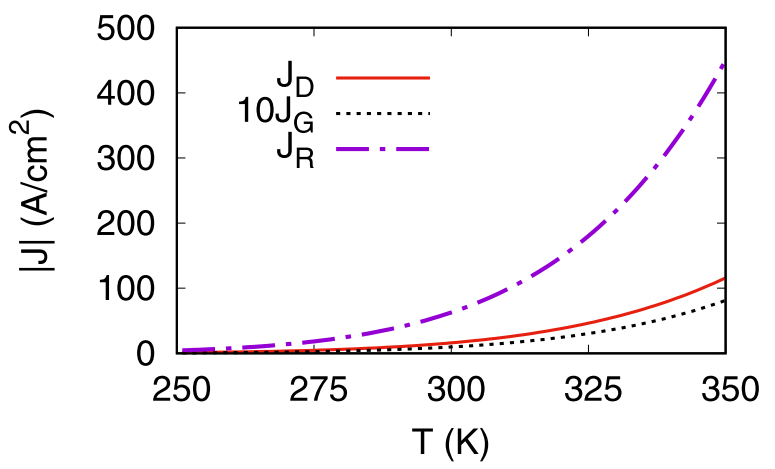

FIG. 1. Temperature dependence of the thermionic current density (absolute value) for conventional materials $\left(J_{R}\right)$, single layer graphene $\left(J_{G}\right)$, and 3D Dirac materials $\left(J_{D}\right)$, where $E_{F}=0.1 \mathrm{eV}$ and $q \phi=0.3 \mathrm{eV}$. In addition, we plot $10 J_{G}$ instead of $J_{G}$ to make it visible. 


$$
\begin{gathered}
I=I_{c}-I_{h}, \\
Q=\left[\left(q \phi+4 k_{B} T_{c}\right) I_{c}-\left(q \phi+4 k_{B} T_{h}\right) I_{h}\right] / q,
\end{gathered}
$$

where $I_{c}=J_{D}\left(\phi, T_{c}\right), I_{h}=J_{D}\left(\phi, T_{h}\right) e^{q V \beta}$, and the positive direction is from the cold side to the hot side.

The average thermal energy per particle is given by

$$
\langle\epsilon\rangle=\hbar v_{F} \frac{\int_{0}^{\infty} k e^{-\alpha k} d k}{\int_{0}^{\infty} e^{-\alpha k} d k},
$$

where $\alpha=\beta \hbar v_{F}$.

For a Dirac system, $\langle\epsilon\rangle$ is found to be $k_{B} T$ for each degree of freedom. This is in contrast to a conventional system where $\langle\epsilon\rangle$ is found to be $\frac{1}{2} k_{B} T$ per degree of freedom. This results in the total average thermal energy for a system with 4 degrees of freedom to be $4 K_{B} T$ for Dirac systems, and the usual $2 K_{B} T$ for conventional parabolic systems.

The voltage and hot side temperature dependence of the energy flow are investigated (shown in Fig. 2). The energy flow is negative when the voltage is absent or small since the energy tends to flow from hot side to cold side. Here, we have set the negative $Q$ to be zero for visual effect (the left corner of Fig. 2). As the voltage increases, $Q$ first increases dramatically and then approaches a constant. The reason is that the voltage only weakens the emission ability of the hot side and keeps the cold side unchanged. Because of this, $Q$ will only be determined by the cold side and will be kept unchanged with the increasing of voltage. The threshold voltage, which changes $Q$ from negative to positive, increases with the hot side temperature due to the increase of the temperature difference between the hot and cold sides. The threshold voltage $\left(V_{c}\right)$ is obtained by setting $Q=0$

$$
\begin{aligned}
V_{c}= & {\left[\frac{q \phi\left(T_{h}-T_{c}\right)}{k_{B} T_{h} T_{c}}+4 \ln \left(\frac{T_{h}}{T_{c}}\right)+\ln \left(\frac{q \phi+2 k_{B} T_{h}}{q \phi+2 k_{B} T_{c}}\right)\right.} \\
& \left.+\ln \left(\frac{q \phi+E_{F}+k_{B} T_{h}}{q \phi+E_{F}+k_{B} T_{c}}\right)\right] \frac{k_{B} T_{h}}{q} .
\end{aligned}
$$

The efficiency $(\eta)$ of the refrigerator is calculated by $\eta=-Q /(I V)$. In order to characterise the performance of

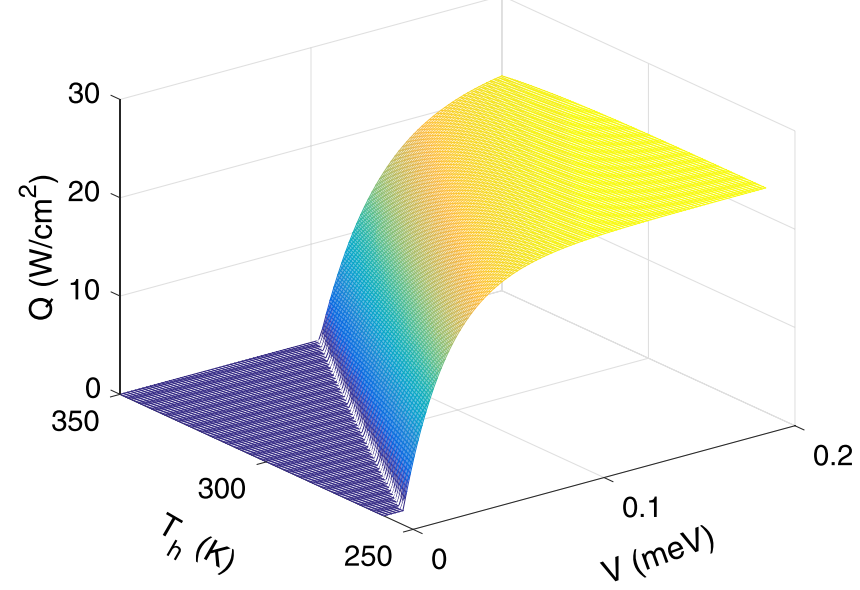

FIG. 2. Voltage and hot side temperature dependence of the energy flow $(Q)$, where $T_{c}=250 \mathrm{~K}, E_{F}=0.1 \mathrm{eV}$, and $q \phi=0.2 \mathrm{eV}$.

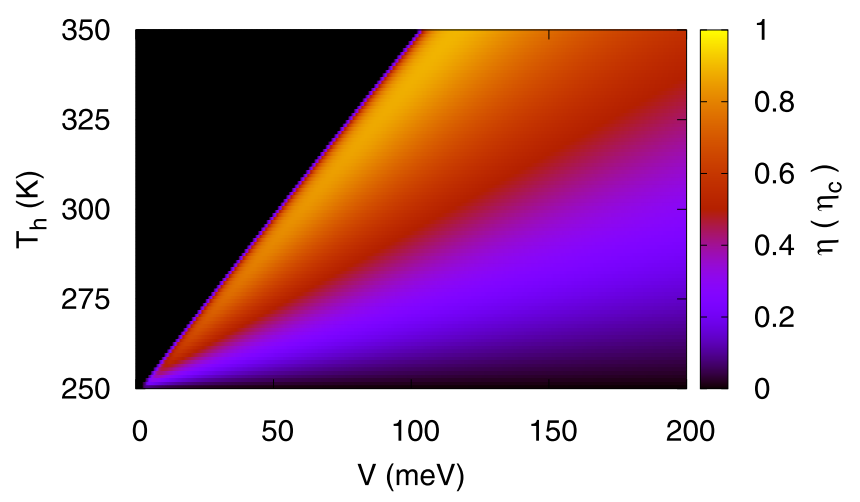

FIG. 3. Voltage and hot side temperature dependence of efficiency $(\eta)$, where $E_{F}=0.1 \mathrm{eV}$ and $q \phi=0.2 \mathrm{eV}$. The color indicates the value of $\eta$ in units of $\eta_{c}$.

the refrigerator, the relative ratio of $\eta$ and the Carnot efficiency $\eta_{c}=T_{c}\left(T_{h}-T_{c}\right)^{-1}$ is calculated as

$$
\frac{\eta}{\eta_{c}}=-\frac{1}{V}\left(\phi+\frac{4 k_{B} T}{q} \times \frac{T_{h}-T_{c} I_{c h}}{1-I_{c h}}\right),
$$

where $I_{c h}=I_{c} / I_{h}$. The voltage and hot side temperature dependence of the efficiency $\eta$ is investigated (shown in Fig. 3). No cooling takes place when the voltage is too low or the hot side temperature is too high (top left corner). As the voltage goes up, the efficiency first increases rapidly to a maximum value and then gradually decreases. The current (I) has the same previously discussed voltage independence at high $\mathrm{V}$ as exhibited by Q. This results in the efficiency gradually decreasing with the voltage after the initial increase, which can also be seen from Eq. (11).

The performance of Dirac materials (labeled by subscript $D$ ) and conventional materials (labeled by subscript $R$ ) in thermionic refrigeration is compared in Fig. 4. Although the energy flow $Q_{R}$ is about four times larger than $Q_{D}$, the efficiency of Dirac materials is considerably high than that of conventional materials. The main reason behind the higher efficiency of 3D Dirac materials is the energy carrying capability of electrons in the different materials. As shown in Eq. (9), the energy carried by Dirac particles per degree of freedom is twice that of a particle with a parabolic energy dispersion. Carriers with a parabolic dispersion have

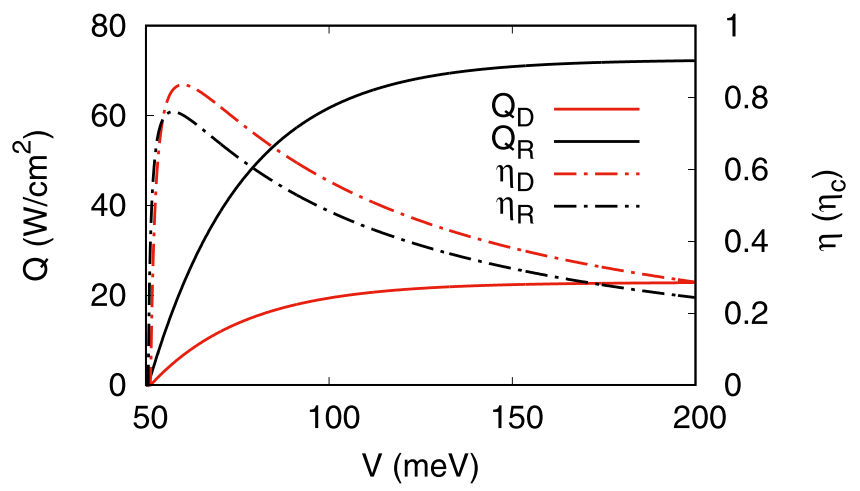

FIG. 4. The voltage dependence of energy flow $Q$ and efficiency $\eta$ of Dirac materials and conventional materials, where the solid line and dashed line are $Q$ and $\eta$, respectively. The red lines are for Dirac materials, and the black lines are for conventional materials. The parameters $T_{c}=250 \mathrm{~K}, T_{h}=300 \mathrm{~K}$, $E_{F}=0.1 \mathrm{eV}$, and $q \phi=0.2 \mathrm{eV}$ are used. 
the highest charge current as shown in Fig. 1. Due to the increase in the average of the thermal energy in 3D Dirac systems, the energy current is enhanced which leads to the system having the greatest thermal efficiency compared to both regular and graphene-based systems. Because of this, considering both their low thermal conductivity and high electron mobility, it is rather reasonable to expect that $3 \mathrm{D}$ Dirac materials should make excellent candidates for thermionic devices. It should be pointed out that we only considered the ideal case of no heat backflow. In a practical device, there is heat backflow due to phonon conduction which is described by $Q_{p}=\left(T_{h}-T_{c}\right) / R_{t h}$, where $R_{t h}$ is the thermal resistance. The heat backflow will significantly reduce the thermal efficiency in all three systems discussed.

In summary, we have shown that 3D Dirac materials exhibit excellent properties with respect to thermionic cooling. Due to an enhanced group velocity along the emission direction, the thermionic current is enhanced compared to graphene. Due to the greater energy carrying capability, the efficiency of thermionic cooling is higher than that in graphene and other conventional materials.

This work was supported by the Australian Research Council Grant (DP160101474).

${ }^{1}$ H. Liu, X. Shi, F. Xu, L. Zhang, W. Zhang, L. Chen, Q. Li, C. Uher, T. Day, and G. J. Snyder, "Copper ion liquid-like thermoelectrics," Nat. Mater. 11(5), 422 (2012).

${ }^{2}$ G. J. Snyder, M. Christensen, E. Nishibori, T. Caillat, and B. Brummerstedt Iversen, "Disordered zinc in $\mathrm{Zn}_{4} \mathrm{Sb}_{3}$ with phonon-glass and electron-crystal thermoelectric properties," Nat. Mater. 3, 458 (2004).

${ }^{3}$ B. Poudel, Q. Hao, Y. Ma, Y. Lan, A. Minnich, B. Yu, X. Yan, D. Wang, A. Muto, D. Vashaee et al., "High-thermoelectric performance of nanostructured bismuth antimony telluride bulk alloys," Science 320(5876), 634-638 (2008).

${ }^{4}$ G. Slack, CRC Handbook of Thermoelectrics, edited by E. M. Rowe (CRC Press, Boca Raton, FL, 1995), Vol. 80, Chap. 18, p. 4016.

${ }^{5}$ X. Shi, J. Yang, J. R. Salvador, M. Chi, J. Y. Cho, H. Wang, S. Bai, J. Yang, W. Zhang, and L. Chen, "Multiple-filled skutterudites: High thermoelectric figure of merit through separately optimizing electrical and thermal transports," J. Am. Chem. Soc. 133(20), 7837-7846 (2011).

${ }^{6}$ G. J. Snyder and E. S. Toberer, "Complex thermoelectric materials," Nat. Mater. 7(2), 105-114 (2008).

${ }^{7}$ K. F. Hsu, S. Loo, F. Guo, W. Chen, J. S. Dyck, C. Uher, T. Hogan, E. Polychroniadis, and M. G. Kanatzidis, "Cubic $\mathrm{AgPb}(\mathrm{m}) \mathrm{SbTe}(2+\mathrm{m})$ : Bulk thermoelectric materials with high figure of merit," Science 303(5659), 818-821 (2004).

${ }^{8}$ M. Zhou, J.-F. Li, and T. Kita, "Nanostructured $\mathrm{AgPb}(\mathrm{m}) \mathrm{SbTe}(\mathrm{m}+2)$ system bulk materials with enhanced thermoelectric performance," J. Am. Chem. Soc. 130(13), 4527-4532 (2008).

${ }^{9}$ K. Biswas, J. He, I. D. Blum, C.-I. Wu, T. P. Hogan, D. N. Seidman, V. P. Dravid, and M. G. Kanatzidis, "High-performance bulk thermoelectrics with all-scale hierarchical architectures," Nature 489(7416), 414 (2012).

${ }^{10}$ W. Xie, J. He, H. J. Kang, X. Tang, S. Zhu, M. Laver, S. Wang, J. R. Copley, C. M. Brown, Q. Zhang et al., "Identifying the specific nanostructures responsible for the high thermoelectric performance of $(\mathrm{Bi}, \mathrm{Sb})_{2} \mathrm{Te}_{3}$ nanocomposites," Nano Lett.10(9), 3283-3289 (2010).

${ }^{11}$ Y. Pei, X. Shi, A. LaLonde, H. Wang, L. Chen, and G. J. Snyder, "Convergence of electronic bands for high performance bulk thermoelectrics," Nature 473(7345), 66 (2011).
${ }^{12}$ Y. Pei, A. D. LaLonde, N. A. Heinz, X. Shi, S. Iwanaga, H. Wang, L. Chen, and G. J. Snyder, "Stabilizing the optimal carrier concentration for high thermoelectric efficiency,” Adv. Mater. 23(47), 5674-5678 (2011).

${ }^{13}$ H. Wang, Y. Pei, A. D. LaLonde, and G. J. Snyder, "Heavily doped p-type $\mathrm{PbSe}$ with high thermoelectric performance: An alternative for PbTe," Adv. Mater. 23(11), 1366-1370 (2011).

${ }^{14}$ W. Liu, X. Tan, K. Yin, H. Liu, X. Tang, J. Shi, Q. Zhang, and C. Uher, "Convergence of conduction bands as a means of enhancing thermoelectric performance of n-type $\mathrm{Mg}_{2} \mathrm{Si}_{1-\mathrm{x}} \mathrm{Sn}_{\mathrm{x}}$ solid solutions," Phys. Rev. Lett. 108(16), 166601 (2012).

${ }^{15}$ H. Wang, Y. Pei, A. D. LaLonde, and G. J. Snyder, "Weak electron-phonon coupling contributing to high thermoelectric performance in n-type PbSe," Proc. Nat. Acad. Sci. U.S.A. 109(25), 9705-9709 (2012).

${ }^{16}$ C. Wood, "Materials for thermoelectric energy conversion," Rep. Prog. Phys. 51, 459-539 (1988).

${ }^{17}$ G. Mahan, "Thermionic refrigeration," J. Appl. Phys. 76(7), 4362-4366 (1994).

${ }^{18}$ A. K. Geim and K. S. Novoselov, "The rise of graphene," Nat. Mater. 6(3), 183-191 (2007).

${ }^{19}$ X.-L. Qi and S.-C. Zhang, "Topological insulators and superconductors," Rev. Mod. Phys. 83(4), 1057 (2011).

${ }^{20}$ Z. Wang, H. Weng, Q. Wu, X. Dai, and Z. Fang, "Three-dimensional Dirac semimetal and quantum transport in $\mathrm{Cd}_{3} \mathrm{As}_{2}$," Phys. Rev. B 88(12), 125427 (2013).

${ }^{21}$ J.-H. Chen, C. Jang, S. Xiao, M. Ishigami, and M. S. Fuhrer, "Intrinsic and extrinsic performance limits of graphene devices on $\mathrm{SiO}_{2}$," Nat. Nanotechnol. 3(4), 206-209 (2008).

${ }^{22}$ Y. S. Ang, Q. Chen, and C. Zhang, "Nonlinear optical response of graphene in terahertz and near-infrared frequency regime," Front. Optoelectron. 8(1), 3-26 (2015).

${ }^{23}$ Q. Chen, Y. Sin Ang, R. Lewis, X. Wang, and C. Zhang, "Photomixing in topological insulator $\mathrm{HgTe} / \mathrm{CdTe}$ quantum wells in terahertz regime," Appl. Phys. Lett. 101(21), 211109 (2012).

${ }^{24}$ F. Zhu, X. Lin, P. Liu, K. Jiang, Y. Wei, Y. Wu, J. Wang, and S. Fan, "Heating graphene to incandescence and the measurement of its work function by the thermionic emission method," Nano Res. 7(4), 553 (2014).

${ }^{25}$ S.-J. Liang and L. Ang, "Electron thermionic emission from graphene and a thermionic energy converter," Phys. Rev. Appl. 3(1), 014002 (2015).

${ }^{26}$ Y. Ang, S.-J. Liang, and L. Ang, "Theoretical modeling of electron emission from graphene," MRS Bull. 42(7), 505-510 (2017).

${ }^{27}$ K. Xu, C. Zeng, Q. Zhang, R. Yan, P. Ye, K. Wang, A. C. Seabaugh, H. G. Xing, J. S. Suehle, C. A. Richter et al., "Direct measurement of Dirac point energy at the graphene/oxide interface," Nano Lett. 13(1), 131-136 (2013).

${ }^{28}$ C. Christodoulou, A. Giannakopoulos, M. Nardi, G. Ligorio, M. Oehzelt, L. Chen, L. Pasquali, M. Timpel, A. Giglia, S. Nannarone et al., "Tuning the work function of graphene-on-quartz with a high weight molecular acceptor," J. Phys. Chem. C 118(9), 4784-4790 (2014).

${ }^{29}$ S.-J. Liang, B. Liu, W. Hu, K. Zhou, and L. Ang, "Thermionic energy conversion based on graphene van der Waals heterostructures," Sci. Rep. 7, 46211 (2017).

${ }^{30}$ M. Neupane, S. Xu, R. Sankar, N. Alidoust, G. Bian, C. Liu, I. Belopolski, T.-R. Chang, H.-T. Jeng, H. Lin et al., "Observation of a three-dimensional topological Dirac semimetal phase in high-mobility $\mathrm{Cd}_{3} \mathrm{As}_{2}$," Nat. Commun. 5, 3786-3786 (2014).

${ }^{31}$ T. Liang, Q. Gibson, M. N. Ali, M. Liu, R. Cava, and N. Ong, "Ultrahigh mobility and giant magnetoresistance in the Dirac semimetal $\mathrm{Cd}_{3} \mathrm{As}_{2}$," Nat. Mater. 14(3), 280-284 (2015).

${ }^{32}$ C. Zhu, F. Wang, Y. Meng, X. Yuan, F. Xiu, H. Luo, Y. Wang, J. Li, X. Lv, L. He et al., "A robust and tuneable mid-infrared optical switch enabled by bulk Dirac fermions,” Nat. Commun. 8, 14111 (2017).

${ }^{33}$ C. Zhang, T. Zhou, S. Liang, J. Cao, X. Yuan, Y. Liu, Y. Shen, Q. Wang, J. Zhao, Z. Yang et al., "Unexpected low thermal conductivity and large power factor in Dirac semimetal $\mathrm{Cd}_{3} \mathrm{As}_{2}$," Chin. Phys. B 25(1), 017202 (2016). 